\title{
Fine mapping of rice drought QTL and study on combined effect of QTL for their physiological parameters under moisture stress condition
}

\author{
K. Baghyalakshmi ${ }^{*}$, P. Jeyaprakash ${ }^{1}$, S. Ramchander ${ }^{1}$, M. Raveendran ${ }^{2}$ and S. Robin ${ }^{1}$ \\ ${ }^{1}$ Centre for Plant Breeding and Genetics, Tamil Nadu Agricultural University, Coimbatore - 641003 (Tamil Nadu), \\ INDIA \\ ${ }^{2}$ Department of Plant Biotechnology, Centre for Plant Molecular Biology and Biotechnology, Tamil Nadu \\ Agricultural University, Coimbatore - 641003 (Tamil Nadu), INDIA \\ *Corresponding author. E-mail: kauverik@gmail.com
}

Received: February 4, 2016; Revised received: August 4, 2016; Accepted: November 3, 2016

\begin{abstract}
The present investigation was undertaken to study the effect of different yield QTL (DTY2.2, DTY $Y_{3.1}$ and $\left.D T Y_{8.1}\right)$ under drought and their physiological response to drought stress. Backcross Inbred Lines (BILs) of IR64 (CB -193 and CB-229) along with IR64, APO and the traditional rice variety Norungan were raised in green house condition under water stress and control to evaluate the effect of the QTL on grain yield. The BIL CB-193 recorded higher photosynthetic rate (22.051), transpiration rate (7.152) and $\mathrm{Ci} / \mathrm{Ca}$ ratio $(0.597)$ whereas the BIL CB-229 recorded high relative water content $(80.76 \%)$. It was found that the combination of three QTL (CB-229) performed better than the susceptible parent and the line with two QTL (CB-193 Fine-mapping of two QTLs viz., qDTY ${ }_{2.2}$ and $q D T Y_{8.1}$, for grain yield (GY) were conducted using backcross derived lines. Composite interval mapping analyses resolved the originally identified $q D T Y_{2.2}$ region of $6.7 \mathrm{cM}$ into a segment of $2.1 \mathrm{cM}$ and two sub QTLs at region between RM23132 and RM1578 (75.75 cM- $77.66 \mathrm{cM})$, RM515 and RM1578 (75.11 cM-77.66 cM) were identified in qDTY 8.1 region. However this study provides a unique opportunity to breeders to introgress such regions together as a unit into high-yielding drought-susceptible varieties through MAS.
\end{abstract}

Keywords: Backcross inbred lines, Drought QTL, Fine mapping, Photosynthetic efficiency, Rice

\section{INTRODUCTION}

Rice (Oryza sativa L.) is the world's most important food crop and a primary food source for about half of the world's population. Rainfed rice occupies $50 \%$ of the total rice area in the world. Over 700 million people depend on rainfed rice grown on 50 million ha in Asia. Rice is semi aquatic plant and hence water loving plant. Drought is the major abiotic stress limiting rice production in these areas. Even a shorter spell of drought during reproductive stage has a greater impact on yield loss. In India, drought is major constraint for rice production and accounts for as much as 15 per cent of yield losses (Markandeya et al., 2005). Varieties with high yield potential and improved drought tolerance could reduce risk and help alleviate poverty in drought-prone rice growing areas. Drought tolerant cultivars such as Apo, Vandana, Way Rarem, Nagina 22, and Adaysel have been shown to out-yield susceptible lines such as IR64 and IR72 by several-fold under upland and lowland drought (Venuprasad et al., 2007; Venuprasad et al., 2009b; Bernier et al., 2007; Vikram et al., 2011; Swamy et al., 2011). Crosses between such tolerant and susceptible lines are useful to identify important QTL underlying variation in grain yield under drought stress.
Molecular tools facilitate the identification and genomic locations of genes controlling traits related to drought tolerance using quantitative trait loci (QTL) analysis. Larger QTL region results in introgression of unwanted segments. Hence to introgress the precise segment the original QTL region has to be fine mapped and size has to be reduced. The present investigation was undertaken to study the effect of different yield QTL $\left(D T Y_{2.2}, D T Y_{3.1}\right.$ and $\left.D T Y_{8.1}\right)$ derived from Apo under drought and their physiological response to drought stress. The two QTLs namely $D T Y_{2.2}$ and $D T Y_{8.1}$ were fine mapped to locate the markers which were close to the QTL region. The original segment of $q D T Y_{3.1}$ is $1.4 \mathrm{cM}$ hence fine mapping was not required (Venuprasad et al., 2009b). Backcross Inbred Lines (BILs) of IR64 (CB-193 and CB-229) along with IR64, APO and the traditional rice variety Norungan were raised in green house condition under water stress and control to evaluate the effect of the QTL on grain yield.

\section{MATERIALS AND METHODS}

Two Backcross Inbred Lines (BILs) of IR64 developed from the cross combination of IR64 X APO along with the parents and Norungan a land race of Tamil Nadu tolerant to drought were used in the investigation. 
APO, drought tolerant upland variety, developed at IRRI, is recommended for cultivation under aerobic conditions. Owing to its drought tolerance nature and good performance under aerobic conditions, they serve as important source for mining drought tolerance QTLs. IR64 is a medium duration and high yielding variety but highly susceptible to drought. A set of 50 BILs from the cross between IR64 X APO were generated which carried three mega QTL classes namely $D T Y_{2.2}, D T Y_{3.1}$ and $D T Y_{8.1}$.

Green house experiment: The two BIL lines of CB$193\left(D_{T Y} Y_{3.1}\right.$ and DTY 8.1$), \mathrm{CB}-229\left(D_{1.2}, D T Y_{3.1}\right.$ and $\left.D T Y_{8.1}\right)$ were raised under control and water stress condition (pot culture experimentation) in four replication during Summer 2015. The seed materials were grown in plastic pots $(30 \mathrm{~cm}$ height $\times 30 \mathrm{~cm}$ diameter with drainage hole) filled with three parts of coir pith and one part of natural clay loam soil. Three plants per pots were maintained and were grown in green house under natural temperature. The crop was irrigated till $45^{\text {th }}$ day and there after irrigation was stopped for two replication (till the Relative Water Content reached 60\%) and the yield parameters were recorded. The positions of the pots were changed frequently to minimize the micro climate effects. Infrared Gas Analyzer (IRGA), a portable photosynthetic system (LICOR- Model LI 6400 version.5) and used for the measurement of different parameters viz., photosynthetic rate, stomatal conductance, transpiration rate and $\mathrm{Ci} / \mathrm{Ca}$ ratio. Relative water content was estimated by Weatherley (1950) method and expressed in percentage. The basic principle (Barrs and Weatherley, 1962) of this technique consists essentially in comparing the water content of leaf tissue when fresh leaf sampled with the fully turgid water content and expressing the results on percentage basis. Several biometrical traits were also observed in both under control and moisture stress condition.

\section{Fine mapping and genotyping}

Generation of genotypic data: Fresh leaves for 50 BILs of the cross IR64xApo were collected and freezedried. DNA was extracted from freeze-dried leaf samples by a modified CTAB (cetyl tri-methyl ammonium bromide) method (Murray and Thompson, 1980) and the DNA was then quantified on $0.8 \%$ agrose gel to adjust the concentration to $25 \mathrm{ng} / \mathrm{LL}^{-1}$. Polymerase chain reaction was performed as described by Panaud et al. (1996). Agrose gel was used for size separation of the amplified DNA fragments using electrophoresis unit (BioRad 96). The DNA fragments were then visualized using trans-illuminator.

Genetic analysis: In this study, rice microsatellite (SSR) markers were used to fine map the previously identified QTL region for grain yield under stress. A total of 13 polymorphic markers for $q D T Y_{2.2}$ and 12 polymorphic markers for $q D T Y_{8.1}$ were added in the originally identified QTL regions in the BILs for fine mapping (Table 4). The markers were taken based on their physical position $(\mathrm{Mb})$ on the indica genome (http:// www.ricetogo.org) and multiplied by 2.7 for approximate estimation of $\mathrm{cM}$ distances for analysis. Composite interval mapping (CIM) was performed using Windows QTL Cartographer 2.5.009 (Wang et al., 2011). The LOD threshold value was obtained empirically from 1,000 permutation tests (Churchill and Doerge, 1994). The LOD thresholds obtained correspond to an experimentwise type I error rate of 0.05 .

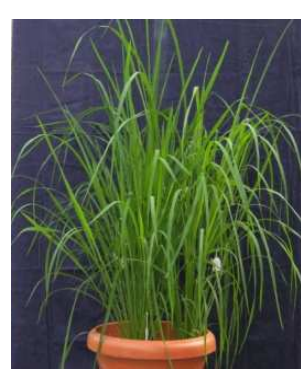

IR $64(C)$

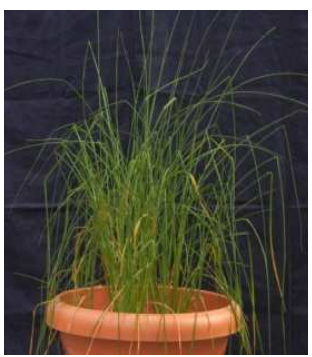

IR $64(S)$

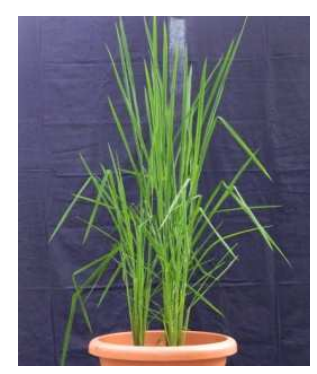

$\mathrm{APO}(\mathrm{C})$

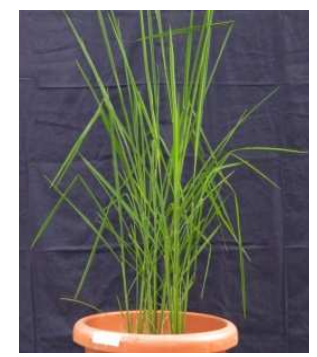

APO (S)

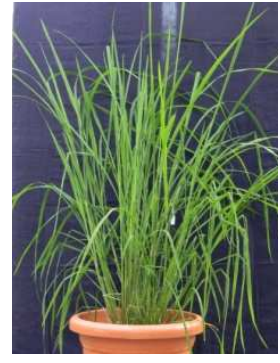

NORUNGAN(C)

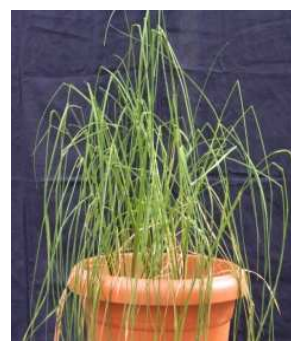

NORUNGAN(S)

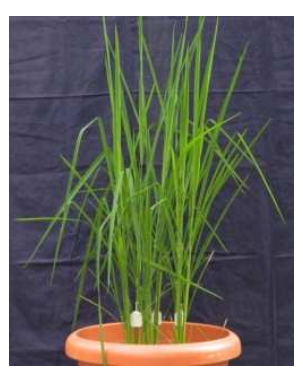

CB 229(C)

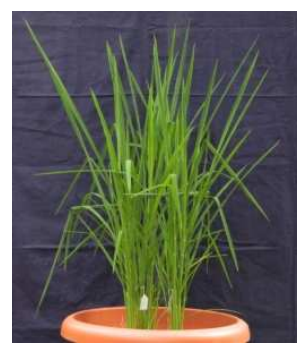

CB 229(S)

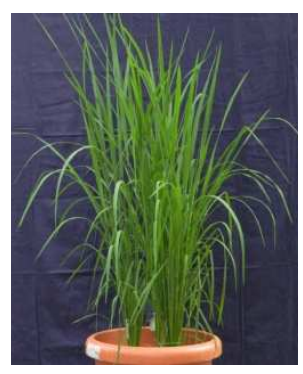

CB 193(C)

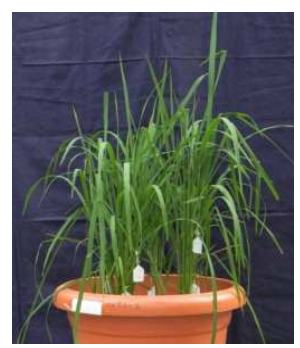

CB 193(S)

Fig. 1. Parents and BILs under irrigated (C-control) and under drought condition (S-Stress). 


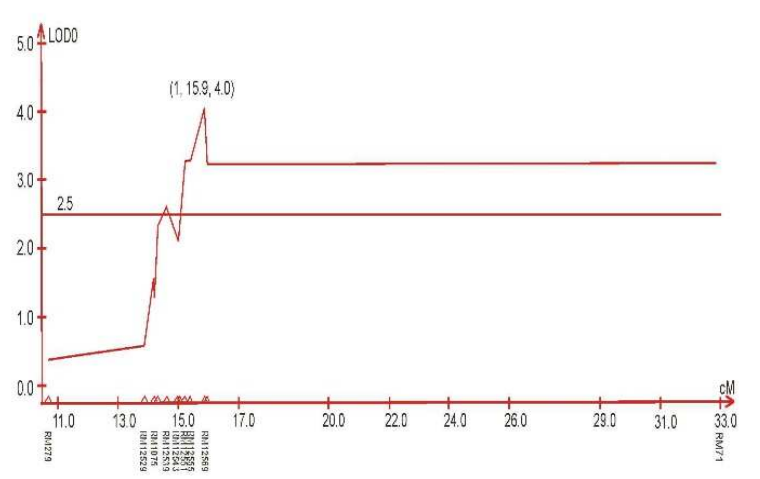

Fig 2a

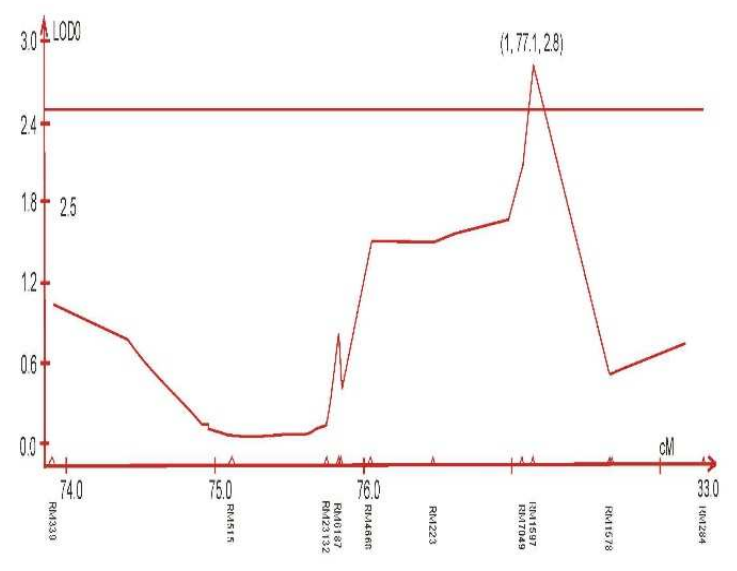

Fig $2 b$.

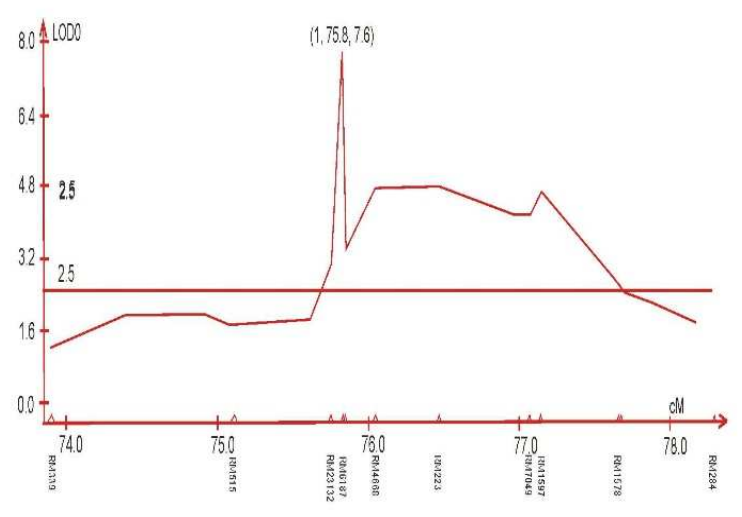

Fig 2c

Fig (a-c). A QTL likelihood curves of LOD score for grain yield $(G Y)$ showing significant regions under severe stress. Genetic distance in $\mathrm{cM}$ between the markers is indicated on $X$ axis. Horizontal lines correspond to critical LOD value. $2 a$ - DTY 2.2 for number of productive tillers under severe drought; $2 b-D T Y 8.1$ for number of productive tillers; $2 c$ DTY 8.1 for panicle length

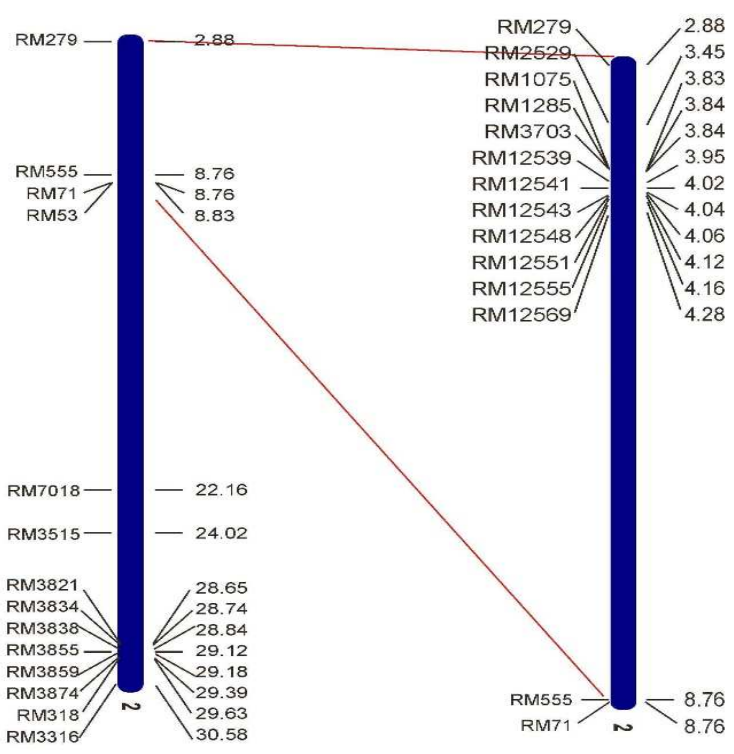

Fig. 3a. Fine mapped region of $q D T Y 2.2$.

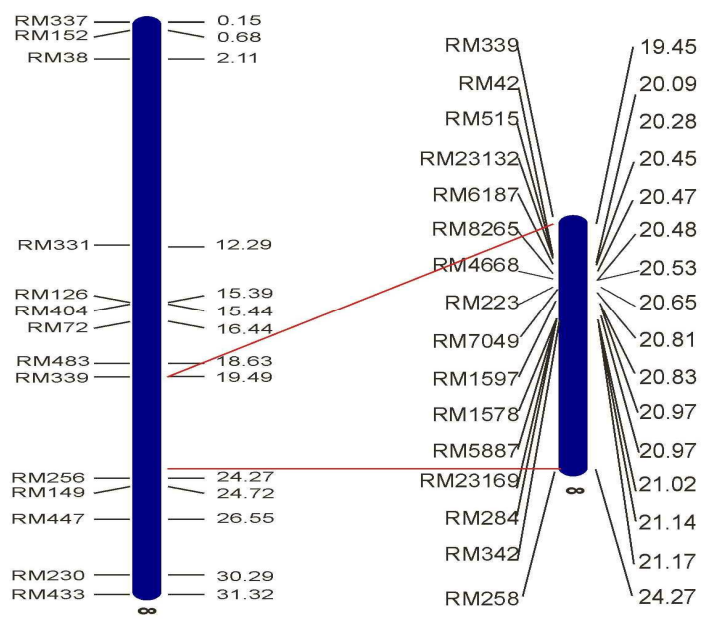

Fig. 3b. Fine mapped region of qDTY8.1.

\section{RESULTS AND DISCUSSION}

Marker assisted selection using consistent yield QTLs under drought could be an alterative approach for improving rice grain yield under drought situations (Swamy et al., 2011; Vikram et al., 2012). QTLs have been identified in the past few years for different drought-related trait through phenotyping and genotyping of large mapping population. Various physiological parmeters viz., photosynthetic rate, stomatal conductance, transpiration rate, $\mathrm{Ci} / \mathrm{Ca}$ ratio and relative water content were measured in BILs namely CB-193 $\left(D T Y_{3.1}\right.$ and $\left.D T Y_{8.1}\right), \mathrm{CB}-229\left(D T Y_{2.2}, D T Y_{3.1}\right.$ and $D T Y_{8.1}$ ), their parents (IR64 and APO) and check (Norungan) at different intervals (52, 58, 66,72 and 78 days after sowing) and the results were presented in Table 1. During initial stage of moisture reduction in pot culture at 52 DAS (days after sowing), the BIL CB 
K. Baghyalakshmi et al. / J. Appl. \& Nat. Sci. 8 (4): 1992-1998 (2016)

Table 1. Estimation of physiological parameters in two Backcross Inbred Lines [CB-193 (DTY ${ }_{3.1}$ and $\left.D T Y_{8.1}\right)$, CB-229 (DTY 2.2 , $D T Y_{3.1}$ and $\left.D T Y_{8.1}\right)$ ] of IR64 both under control and water stress condition (pot culture).

\begin{tabular}{|c|c|c|c|c|c|c|c|c|c|c|c|c|}
\hline \multirow{2}{*}{$\begin{array}{l}\text { Genotypes } \\
52 \text { DAS } \\
\end{array}$} & \multicolumn{2}{|c|}{ Soil Moisture (\%) } & \multicolumn{2}{|c|}{$\begin{array}{l}\text { Photosynthetic rate }(\mu \\
\left.\text { mol CO} \mathrm{Cm}_{2} \mathrm{~m}^{-1}\right)\end{array}$} & \multicolumn{2}{|c|}{$\begin{array}{c}\text { Stomatal Conductance } \\
\left(\mathrm{mol} \mathrm{H}_{2} \mathrm{O} \mathrm{m}^{-2} \mathrm{~s}^{-1}\right)\end{array}$} & \multicolumn{2}{|c|}{$\begin{array}{l}\text { Transpiration Rate } \\
\left(\mathbf{m m o l ~ H _ { 2 }} \mathbf{O ~ m}^{-2} \mathbf{s}^{-1}\right)\end{array}$} & \multicolumn{2}{|c|}{$\mathrm{Ci} / \mathrm{Ca}$} & \multicolumn{2}{|c|}{$\begin{array}{c}\text { Relative Water } \\
\text { Content }(\%)\end{array}$} \\
\hline & C & $\mathbf{S}$ & C & $\mathbf{S}$ & C & $\mathbf{S}$ & C & $\mathbf{S}$ & C & $\mathbf{S}$ & C & $\mathbf{S}$ \\
\hline CB-193 (2QTL) & 96.43 & 65.56 & 23.260 & $22.051^{*}$ & 0.255 & $0.270^{*}$ & 7.468 & $7.152^{*}$ & 0.559 & $\begin{array}{l}0.597 \\
* \\
0.591\end{array}$ & 98.59 & 95.72 \\
\hline CB-229 (3QTL) & 89.52 & 64.09 & $28.88^{*}$ & 20.942 & $0.350 *$ & $0.252^{*}$ & $9.325^{*}$ & 6.660 & $0.589 *$ & $\begin{array}{l}* \\
0.606\end{array}$ & 98.11 & $97.65^{*}$ \\
\hline Norungan & 90.82 & 65.76 & 21.138 & $21.543^{*}$ & 0.259 & $0.271^{*}$ & 7.117 & $7.182^{*}$ & 0.598 & $*$ & 99.36 & 97.32 \\
\hline $\mathrm{APO}$ & 89.39 & 62.10 & 20.829 & 20.090 & 0.241 & 0.176 & 6.751 & 5.005 & $0.578^{*}$ & 0.472 & 99.05 & $98.31 *$ \\
\hline IR64 & 91.73 & 68.19 & 22.821 & 18.107 & 0.217 & 0.142 & 6.084 & 6.478 & 0.495 & 0.397 & 96.55 & 96.52 \\
\hline MEAN & 91.58 & 65.14 & 23.39 & 20.55 & 0.26 & 0.22 & 7.35 & 6.50 & 0.56 & 0.53 & 98.33 & 97.10 \\
\hline $\begin{array}{l}\text { SE } \\
58 \text { DAS }\end{array}$ & 1.29 & 1.00 & 1.45 & 0.69 & 0.02 & 0.03 & 0.54 & 0.40 & 0.02 & 0.04 & 0.49 & 0.45 \\
\hline $\begin{array}{l}\text { CB-193 } \\
\text { (2QTL) } \\
\text { CB-229 }\end{array}$ & 96.43 & 65.56 & $\begin{array}{l}32.856 \\
*\end{array}$ & 17.382 & $0.474 *$ & $0.247^{*}$ & $\begin{array}{l}16.083 \\
*\end{array}$ & $6.519^{*}$ & $0.627 *$ & $\begin{array}{l}0.653 \\
*\end{array}$ & $98.59^{*}$ & 95.72 \\
\hline (3QTL) & 78.68 & 61.53 & 25.761 & $20.025^{*}$ & 0.293 & 0.108 & 12.098 & 4.473 & 0.555 & 0.178 & $98.86^{*}$ & $97.22 *$ \\
\hline Norungan & 80.98 & 60.05 & 20.928 & $20.432^{*}$ & 0.201 & 0.157 & 8.826 & $6.922^{*}$ & 0.496 & $\begin{array}{l}0.384 \\
0.641\end{array}$ & 97.02 & $97.00 *$ \\
\hline APO & 83.78 & 54.91 & 23.302 & 17.190 & 0.248 & $0.236^{*}$ & 10.374 & 6.288 & 0.531 & $*$ & 97.88 & 96.09 \\
\hline IR64 & 81.16 & 62.85 & 21.817 & 16.293 & 0.284 & 0.184 & 11.297 & 5.534 & $0.599 *$ & 0.574 & 97.56 & 95.60 \\
\hline MEAN & 84.21 & 60.98 & 24.93 & 18.26 & 0.30 & 0.19 & 11.74 & 5.95 & 0.56 & 0.49 & 97.98 & 96.33 \\
\hline $\begin{array}{l}\text { SE } \\
66 \text { DAS }\end{array}$ & 3.16 & 1.77 & 2.14 & 0.83 & 0.05 & 0.03 & 1.22 & 0.43 & 0.02 & 0.09 & 0.34 & 0.33 \\
\hline $\begin{array}{l}\text { CB-193 } \\
\text { (2QTL) } \\
\text { CB-229 }\end{array}$ & 91.74 & 41.01 & $\begin{array}{l}31.614 \\
* \\
32.918\end{array}$ & 15.527 & $0.810^{*}$ & $\begin{array}{l}0.090 \\
0.196\end{array}$ & $\begin{array}{l}13.700 \\
*\end{array}$ & 3.807 & 0.776 & $\begin{array}{l}0.219 \\
0.534\end{array}$ & 97.11 & 88.86 \\
\hline (3QTL) & 88.03 & 43.52 & $*$ & $19.751^{*}$ & 0.732 & $*$ & 12.768 & $4.389^{*}$ & 0.750 & $*$ & 93.42 & $91.53 *$ \\
\hline Norungan & 69.55 & 39.69 & 27.755 & $17.391 *$ & 0.703 & 0.141 & 12.051 & 3.323 & 0.780 & 0.441 & $97.57 *$ & 86.32 \\
\hline $\mathrm{APO}$ & 74.09 & 36.04 & 25.086 & 14.852 & 0.657 & 0.112 & 11.360 & $4.604^{*}$ & 0.787 & $\begin{array}{l}0.392 \\
0.581\end{array}$ & $97.35^{*}$ & 87.74 \\
\hline IR64 & 90.30 & 49.93 & 26.577 & 13.332 & $0.862 *$ & 0.151 & 12.950 & $4.499^{*}$ & 0.820 & $*$ & 97.25 & 88.30 \\
\hline MEAN & 82.74 & 42.04 & 28.79 & 16.17 & 0.75 & 0.14 & 12.57 & 4.12 & 0.78 & 0.43 & 96.54 & 88.55 \\
\hline $\begin{array}{l}\text { SE } \\
72 \text { DAS }\end{array}$ & 4.55 & 2.31 & 1.50 & 1.11 & 0.04 & 0.02 & 0.40 & 0.24 & 0.01 & 0.06 & 0.78 & 0.86 \\
\hline $\begin{array}{l}\text { CB-193 } \\
\text { (2QTL) } \\
\text { CB-229 }\end{array}$ & 87.25 & 17.95 & $\begin{array}{l}30.938 \\
32.672\end{array}$ & $\begin{array}{l}15.465 \\
* \\
16.657\end{array}$ & $0.642^{*}$ & $\begin{array}{l}0.141 \\
*\end{array}$ & $\begin{array}{l}14.695 \\
* \\
15.167\end{array}$ & 3.224 & $0.734^{*}$ & $\begin{array}{l}0.502 \\
*\end{array}$ & $96.20 *$ & 82.52 \\
\hline (3QTL) & 79.08 & 27.49 & $\begin{array}{l}* \\
32.778\end{array}$ & $*$ & $0.608^{*}$ & $\begin{array}{l}0.074 \\
0.151\end{array}$ & $*$ & 3.072 & $0.702 *$ & $\begin{array}{l}0.033 \\
0.581\end{array}$ & 90.64 & $88.41^{*}$ \\
\hline Norungan & 75.21 & 16.28 & $*$ & 13.332 & 0.498 & $*$ & 13.342 & $4.499^{*}$ & 0.648 & $*$ & $97.54 *$ & 79.18 \\
\hline $\mathrm{APO}$ & 78.94 & 30.50 & 28.294 & 14.119 & 0.451 & 0.113 & $\begin{array}{l}12.776 \\
14.657\end{array}$ & 2.803 & 0.669 & 0.439 & 92.15 & $86.38 *$ \\
\hline IR64 & 80.10 & 34.16 & 29.375 & 12.352 & 0.539 & 0.066 & $*$ & 2.960 & 0.700 & 0.177 & 94.83 & 79.71 \\
\hline MEAN & 87.25 & 25.28 & 30.81 & 14.39 & 0.55 & 0.11 & 14.13 & 3.31 & 0.69 & 0.35 & 94.27 & 83.24 \\
\hline $\begin{array}{l}\text { SE } \\
77 \text { DAS }\end{array}$ & 1.96 & 3.50 & 0.89 & 0.76 & 0.03 & 0.02 & 0.45 & 0.30 & 0.01 & 0.10 & 1.27 & 1.82 \\
\hline $\begin{array}{l}\text { CB-193 } \\
\text { (2QTL) } \\
\text { CB-229 }\end{array}$ & 85.02 & 16.22 & 19.978 & $10.286^{*}$ & 0.331 & $\begin{array}{l}0.110 \\
*\end{array}$ & $\begin{array}{l}11.514 \\
*\end{array}$ & $4.102^{*}$ & $0.678 *$ & $\begin{array}{l}0.556 \\
*\end{array}$ & 98.99 & 72.41 \\
\hline (3QTL) & 71.96 & 22.87 & $\begin{array}{l}18.424 \\
26.040\end{array}$ & $10.473^{*}$ & 0.268 & 0.066 & $\begin{array}{l}9.801 \\
11.941\end{array}$ & 2.678 & 0.646 & 0.294 & $99.38 *$ & $80.76^{*}$ \\
\hline Norungan & 70.32 & 16.89 & $*$ & 4.685 & $0.363^{*}$ & $\begin{array}{l}0.032 \\
0.131\end{array}$ & $\begin{array}{l}* \\
12.213\end{array}$ & 1.337 & 0.632 & $\begin{array}{l}0.351 \\
0.597\end{array}$ & 98.19 & 63.52 \\
\hline APO & 66.68 & 23.44 & 20.974 & $10.951^{*}$ & $0.373^{*}$ & $*$ & $*$ & $4.923^{*}$ & $0.698^{*}$ & $*$ & $99.36^{*}$ & $78.10^{*}$ \\
\hline IR64 & 75.39 & 27.65 & 17.937 & 6.851 & 0.238 & 0.047 & 8.336 & 1.935 & 0.620 & 0.344 & 99.19 & 66.93 \\
\hline MEAN & 73.87 & 21.41 & 20.67 & 8.65 & 0.31 & 0.08 & 10.76 & 2.99 & 0.65 & 0.43 & 99.02 & 72.34 \\
\hline SE & 3.12 & 2.15 & 1.45 & 1.23 & 0.03 & 0.02 & 0.74 & 0.67 & 0.01 & 0.06 & 0.22 & 3.25 \\
\hline
\end{tabular}

C - Control, S - Moisture Stress, DAS - days after sowing

-193 recorded higher photosynthetic rate (22.051), stomatal conductance $(0.270)$, transpiration rate $(7.152)$ and $\mathrm{Ci} / \mathrm{Ca}$ ratio $(0.597)$ whereas the BIL CB-229 recorded high relative water content $(\%)$. The plants started expressing leaf rolling symptoms at 21 days after drought induction (Fig. 1). When moisture stress increased during 58, 66, 72 and 78 DAS, the line CB229 registered higher gas exchange parameters espe- 
Table 2a. Yield attributing traits of Backcross Inbred Lines [CB-193 (DTY 3.1 and DTY $\left.{ }_{8.1}\right), \mathrm{CB}-229\left(D T Y_{2.2}, D T Y_{3.1}\right.$ and $\left.D T Y_{8.1}\right)$ ] of IR64 both under control and water stress condition (pot culture). Define c and S below each table.

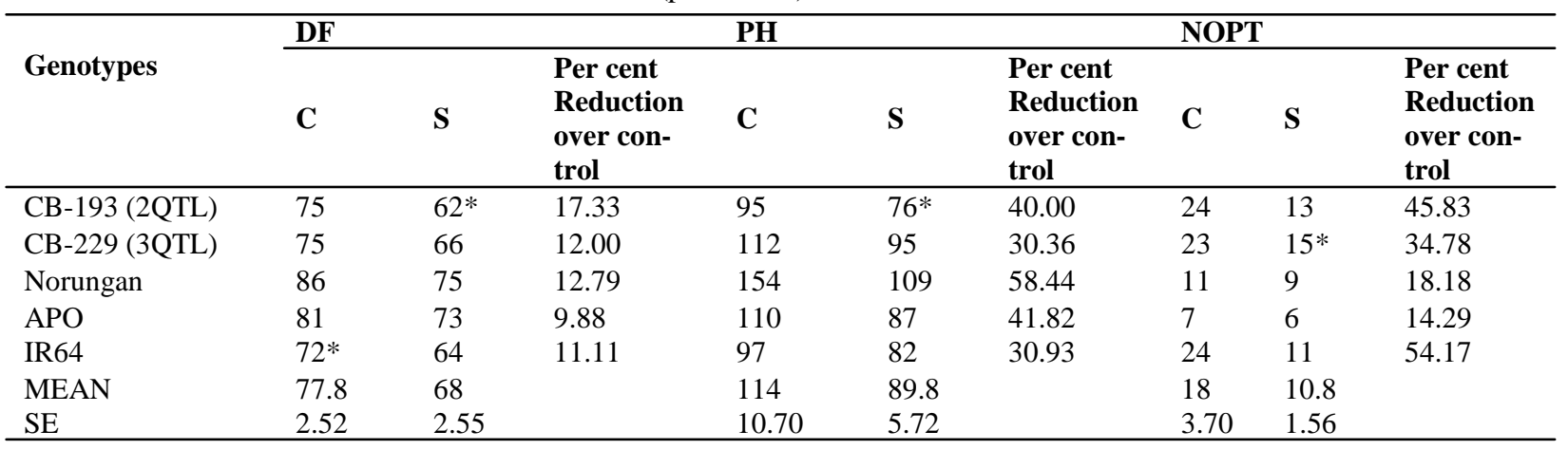

C - Control, S - Moisture Stress

Table 2b. Yield attributing traits of Backcross Inbred Lines [CB-193 $\left(D T Y_{3.1}\right.$ and $\left.D T Y_{8.1}\right)$, CB-229 $\left(D T Y_{2.2}, D T Y_{3.1}\right.$ and $\left.\left.D T Y_{8.1}\right)\right]$ of IR64 both under control and water stress condition (pot culture).

\begin{tabular}{|c|c|c|c|c|c|c|c|c|c|c|c|c|}
\hline \multirow[b]{2}{*}{$\begin{array}{l}\text { Geno- } \\
\text { types }\end{array}$} & \multicolumn{2}{|l|}{ NFG } & \multicolumn{3}{|c|}{ SPF } & \multicolumn{3}{|c|}{ HGW } & \multicolumn{3}{|c|}{ SPY } & \multirow[b]{2}{*}{$\begin{array}{l}\text { Per cent } \\
\text { reduc- } \\
\text { tion } \\
\text { over } \\
\text { control }\end{array}$} \\
\hline & C & $\mathbf{S}$ & $\begin{array}{l}\text { Per cent } \\
\text { reduc- } \\
\text { tion } \\
\text { over } \\
\text { control }\end{array}$ & C & $\mathbf{S}$ & $\begin{array}{l}\text { Per cent } \\
\text { reduc- } \\
\text { tion } \\
\text { over } \\
\text { control }\end{array}$ & C & $\mathbf{S}$ & $\begin{array}{l}\text { Per cent } \\
\text { reduc- } \\
\text { tion } \\
\text { over } \\
\text { control }\end{array}$ & C & $\mathbf{S}$ & \\
\hline CB-193 & & & & & & & 2.6 & & & 15.2 & & \\
\hline (2QTL) & 165 & 118 & 28.48 & $92.7 *$ & 66.3 & 28.48 & 2 & 2.41 & 8.02 & 3 & 7.36 & 51.67 \\
\hline CB-229 & & & & & & & 2.6 & & & 19.0 & & \\
\hline (3QTL) & $274 *$ & 140 & 48.91 & 89.3 & 66 & 26.09 & 4 & 2.47 & 6.44 & $9^{*}$ & $9.46^{*}$ & 50.44 \\
\hline & & & & & & & 2.7 & 2.68 & & & & \\
\hline Norungan & 172 & $\begin{array}{l}136 \\
172\end{array}$ & 20.93 & 89.1 & 66.7 & 25.14 & 3 & $*$ & 1.83 & 6.93 & 4.56 & 34.20 \\
\hline APO & $259^{*}$ & $*$ & 33.59 & 91.5 & 71.4 & 21.97 & $\begin{array}{l}2.3 \\
2.8\end{array}$ & 2.25 & 2.17 & $\begin{array}{l}7.87 \\
16.3\end{array}$ & 4.91 & 37.61 \\
\hline IR64 & 157 & $\begin{array}{l}86 \\
130 .\end{array}$ & 45.22 & 89.2 & $\begin{array}{l}47.5 \\
63.5\end{array}$ & 46.75 & $\begin{array}{l}2 \\
2.6\end{array}$ & 2.24 & 20.57 & 7 & 4.3 & 73.73 \\
\hline MEAN & 205.4 & $\begin{array}{l}4 \\
14.1\end{array}$ & & 90.36 & 8 & & $\begin{array}{l}22 \\
0.0\end{array}$ & 2.41 & & $\begin{array}{l}13.1 \\
2.41\end{array}$ & 5.72 & \\
\hline SE & 25.17 & 0 & & 0.74 & 4.14 & & 9 & 0.10 & & 4 & 0.77 & \\
\hline
\end{tabular}

C - Control, S - Moisture Stress; DF-days to $50 \%$ flowering, PH- plant height, NOPT- number of productive tillers, RL- root length, NFG- number of filled grains, SPF- spiklet fertility, HGW- hundred grain weight, SPY- single plant yield.

cially photosynthetic rate and relative water content as resistant parent APO and check Norungan. This clearly revealed that, the BIL line CB-229 maintained higher internal water status with normal physiological process of photosyntheis, stomatal conductance and transpiration rate and withstand under higher moister stress conditions. Under stress condition, the population varied widely for all the physiological traits studied viz., photosynthetic rate, transpiration rate, stomatal conductance and relative water content. Apo had higher stomatal conductance when compared to IR64 and the BILs also had higher stomatal conductance than IR64. The same kind of results was obtained by Tezera et al. (2002) who reported that higher stomatal conductance would result in higher photosynthetic rate and biomass production. Martinez et al. (2007) also pointed out that higher stomatal conductance may be an enhanced adaptation of plants to drought environments. Chen et al. (1995) observed that elevating photosynthetic rate is beneficial to dry matter production and yield. Cao et al. (2001) reported that photosynthetic rate among rice varieties were significant and suggested the net photosynthetic rate as a selection parameter for drought resistant genotypes. The similar result was obtained from this study also and Apo had more photosynthetic rate along with BILs when compared to IR64. Araus et al. (2002) reported that higher yielding genotypes under drought had greater stomatal conductance and transpiration rate. Sikuku et al. (2010) observed transpiration rate in NERICA rice varieties generally decreased with increase in soil water deficit.

Fine mapping: Fine mapping of $q D T Y_{2.2}$ and $q D T Y_{8.1}$ was done under severe stress wherein the yield traits were recorded. The drought QTL $q D T Y_{3.1}$ was not fine mapped in this study since the closest linked marker was already reported by Venuprasad et al., 2009a. CIM analysis of markers within $q D T Y_{2.2}$ showed a region between RM12529 and RM12571 (13.87cM - 
Table 3. Fine-mapped regions within different QTLs for grain yield under stress.

\begin{tabular}{|c|c|c|c|c|c|c|c|}
\hline $\begin{array}{l}\text { QTL-Fine mapped } \\
\text { region }\end{array}$ & $\begin{array}{l}\text { Associated trait } \\
\text { under stress }\end{array}$ & Marker Inerval & Peak position & $\begin{array}{l}\text { Marker closest } \\
\text { to the peak }\end{array}$ & $\mathbf{R}^{2}(\%)$ & Add\% & $\begin{array}{l}\text { LOD } \\
\text { score }\end{array}$ \\
\hline$q D T Y 2.2$ & Productive tillers & $\begin{array}{l}\text { RM12529- } \\
\text { RM12571 }\end{array}$ & $15.9 \mathrm{cM}$ & RM12569 & 19.0 & 28.5 & 4 \\
\hline qDTY8.1 & Productive tillers & $\begin{array}{l}\text { RM23132- } \\
\text { RM1578 }\end{array}$ & $77.1 \mathrm{cM}$ & RM1597 & 23.0 & 15.3 & 2.9 \\
\hline$q D T Y 8.1$ & Panicle length & $\begin{array}{l}\text { RM515- } \\
\text { RM1578 }\end{array}$ & $75.8 \mathrm{cM}$ & RM6187 & 43.0 & 42.8 & 7.6 \\
\hline
\end{tabular}

Table 4. List of polymorphic markers used for fine mapping.

\begin{tabular}{cccc}
\hline \multicolumn{2}{c}{ Chromosome 2 } & \multicolumn{2}{c}{ Chromosome 8 } \\
\hline Markers & $\begin{array}{c}\text { Position } \\
\text { (cM) }\end{array}$ & Markers & $\begin{array}{c}\text { Position } \\
\text { (cM) }\end{array}$ \\
\hline RM279 & 10.67 & RM339 & 73.9 \\
RM12529 & 13.87 & RM515 & 75.11 \\
RM1075 & 14.20 & RM23132 & 75.75 \\
RM1285 & 14.21 & RM6187 & 75.83 \\
RM3703 & 14.31 & RM8265 & 75.85 \\
RM12539 & 14.61 & RM4668 & 76.05 \\
RM12543 & 14.98 & RM223 & 76.47 \\
RM12548 & 15.05 & RM7049 & 77.07 \\
RM12551 & 15.21 & RM1597 & 77.14 \\
RM12555 & 15.39 & RM1578 & 77.66 \\
RM12569 & 15.86 & RM5887 & 77.67 \\
RM12571 & 15.95 & RM284 & 78.29 \\
RM71 & 33.0 & & \\
\hline
\end{tabular}

$15.95 \mathrm{cM}$ ) having an effect on GY under drought stress condition. The peak was detected at $15.9 \mathrm{cM}$ under stress conditions with RM12569 as the closest marker to the peak at LOD 4 (Fig. 2a and 3a). The QTL explained a phenotypic variance of $19.0 \%$ and had an additive effect of 28.5 (Table 3). Similarly analysis of $q D T Y_{8.1}$ exhibited two sub QTLs at region between RM23132 and RM1578 (75.75 cM- $77.66 \mathrm{cM})$, RM515 and RM1578 (75.11 cM-77.66 cM) for the traits viz., panicle length and number of productive tillers under drought conditions with RM1597 and RM6187 as the closest markers to the peak respectively (Fig 2 b and $2 c \& 3 b$ ). The peak was detected at 77.1 and $75.8 \mathrm{cM}$ under stress conditions with LOD of 2.9 and 7.6 respectively. The QTL explained a phenotypic variance of $23.1 \%$ and $43 \%$ and had an additive effect of 15.3 and 42.8 (Table 3) under drought condition.

In the present investgation, several biometrical traits were observed in all genotypes under study which exhibited that, the BIL CB-229 had recorded higher number of productive tillers, root length, number of filled grains per panicle, spikelet fertility percentage, hundred grain weight and grain yield under moisture stress condition when compared to the BIL CB-193. This could be due to the presence of two QTL regions together within $q D T Y_{8.1}$ showing larger effect in drought stress condition. However, $q D T Y_{2.2}, q D T Y_{3.1}$ and $q D T Y_{8.1}$ have shown a high and consistent additive effect under severe drought condition. The $q D T Y_{2.2}$ exhibited higher effect on increased number of tillers and $q D T Y_{8.1}$ had an effect on panicle length and number of productive tillers thus increasing the yield under drought. Since, the QTL $D T Y_{2.2}$ was responsible for root length (MacMillan et al., 2006; Kamoshita et al., 2002) and root thickness (Champoux et al., 1995, Dixit et al., 2012). In this study also, the BIL CB-229 possessing QTL $D T Y_{8.1}$ was associated with grain yield under stress condition. The similar result was already reported by Vikram et al. (2012). But the significant advantage of CB-193 was earliness in flowering and reduced plant height. Both the BILs of IR64-193 $\left(D T Y_{3.1}\right.$ and $\left.D T Y_{8.1}\right)$ and IR64-229 $\left(D T Y_{2.2}, D T Y_{3.1}\right.$ and $\left.D T Y_{8.1}\right)$ were earlier in flowering with reduced plant height, which possessed $D T Y_{3.1}$ responsible for flowering date and plant height (Venuprasad et al., 2009a). Ding et al. (2011) reported complex nature of large-effect QTLs for stress tolerance working on a QTL on chromosome 4 controlling root volume per tiller co-segregating with flag -leaf width and spikelet number per panicle.

\section{Conclusion}

The present investigation was undertaken to study the effect of different yield QTL $\left(D T Y_{2.2}, D T Y_{3.1}\right.$ and $\left.D T Y_{8.1}\right)$ derived from Apo under drought and their physiological response to drought stress. The $q D T Y_{2.2}$ showed larger effect under drought but a very low effect under moderate stress. The other two qDTY (3.1 and 8.1) showed its effect only under severe drought stress. It should be possible to exploit these fine mapped QTL after development of gene-based markers, for improving grain yield of valuable varieties such as IR64 in stress prone environments via marker assisted backcross breeding. In this study an attempt was made to narrow down the originally identified QTL regions so as to introgress precisely the smallest possible segment of these QTLs showing profound effect on grain yield under drought while minimizing the chances of introgression of any undesirable linked trait. The successful introgression of the fine-mapped regions of these QTLs by MAS provides an opportunity to improve the drought tolerance of well-adapted, highyielding but drought-susceptible popular rice varieties.

\section{ACKNOWLEDGEMENTS}

Authors profoundly acknowledge The Department of Biotechnology for funding the scheme "Comparative transcriptomics between rice and resurrection plants and exploitation of novel genes for dehydration tolerance for 
improvement of drought tolerance in rice (Indo- Australia)" and successful completion of the research work.

\section{REFERENCES}

Araus, J.L., Slafer, G.A., Reynolds, M.P. and Royo, C. (2002). Plant breeding and drought in C3 cereals: what should we breed for. Ann. Bot., 89 : 925-940

Barrs, H.D. and Weatherley. P.E. (1962). A reexamination of the relative turgidity technique for estimating water deficits in leaves. Aust. J. Biol. Sci., 15:415-428

Bernier, J., Kumar, A., Ramaiah, V., Spaner, D. and Atlin, G. (2007). A large-effect QTL for grain yield under reproductive-stage drought stress in upland rice. Crop Sci., 47: 507518

Cao, S.Q., Zhai, H.Q., Yang, T.N., Zhang R.X. and Kuang, T.Y. (2001). Studies on photosynthetic rate and function duration of rice germplasm. Chinese J. Rice Sci., 15(1): 29-34

Champoux, M.C., Wang, G., Sarkarung, S., Mackill, D.J., O’Toole, J.C., Huang, N., McCouch S.R. (1995). Locating genes associated for root morphology and drought avoidance in rice via linkage to molecular markers. Theor. Appl. Genet., 90: 969-981

Chen, W.F., Xu, Z.J. and Zhang, B.L. (1995). Physiological bases of super high yield breeding in rice. Liao Ning Science and Technology Publishing Company, Shenyang, China.

Churchill, G.A. and Doerge, R.W. (1994). Empirical threshold values for quantitative trait mapping. Genetics., 138:963971

Ding, X., Li, X. and Xiong, L. (2011). Evaluation of nearisogenic lines for drought resistance QTL and fine mapping of a locus affecting flag leaf width, spikelet number, and root volume in rice. Theor. Appl. Genet., 123:815-826

Dixit S., Mallikarjuna Swamy B.P., Prashant Vikram, Ahmed, H.U., Sta Curz, M.T., Modesto Amante, Dinesh Atri, Hei Leung and Aravind Kumar. (2012). Fine mapping of QTLs for rice grain yield under drought reveals sub-QTLs conferring a respose to variable drought severities. Theor. Appl. Genet., 125: 155-169

Kamoshita, A, Wade, L.J., Ali, M.L., Pathan, M.S., Zhang, J., Sarkarung, S. and Nguyen, H.T. (2002). Mapping QTLs for root morphology of a rice population adapted to rainfed lowland conditions. Theor. Appl. Genet., 104:880-893

MacMillan, K., Emrich, K., Piepho, H.P., Mullins, C.E. and Price, A.H. (2006). Assessing the importance of genotype 9 environmental interaction for root traits in rice using a mapping population. II.Conventional QTL analysis. Theor. Appl. Genet., 113:953-964

Markandeya, G., Babu, P.R. and Lachagari, V.B.R. (2005). Functional genomics of drought stress response in rice: transcript mapping of annotated unigenes of an indica rice (Oryza sativa L. cv. Nagina 22). Curr. Sci., 89:496-514

Martinez, J.P., Silva, H., Ledent, J.F. and Pinto, M. (2007).
Effect of drought stress on the osmotic adjustment, cell wall elasticity and cell volume of six cultivars of common beans (Phaseolus vulgaris L.). Euro. J. Agro., 26: 30-38

Murray, M.G. and Thompson, W.F. (1980). Rapid isolation of high molecular weight plant DNA. Nucleic Acid Res., 8, 4321-4326

Panaud, O., Chen, X., McCouch, S. (1996). Development of microsatellite markers and characterization of simple sequence length polymorphism (SSLP) in rice (Oryza sativa L.). Mol. Gen. Genet., 252:597-607

Sikuku, P.A., Netondo, G.W., Onyango, J.C. and Musyimi, D. M. (2010). Effects of water deficit on physiology and Morphology of three varieties of NERICA Rainfed rice (Oryza sativa L.). ARPN Journal of Agricultural and Biological Science, 5: 1

Swamy, B.P.M., Vikram, P., Dixit, S., Ahmed, H.U. and Kumar, A. (2011). Metaanalysis of grain yield QTL identified during agricultural drought in grasses showed consensus. BMC Genomics., 12:319

Tezara, W., Mitchel, V., Driscul, S.P. and Lawlor, D.W. (2002). Effects of water deficit and its interaction with $\mathrm{CO} 2$ supply on the biochemistry and physiology of photosynthesis in sunflower. J. Exp. Bot., 53: 1781-1791

Venuprasad, R., Lafitte, H.R. and Atlin, G.N. (2007). Response to direct selection for grain yield under drought stress in rice. Crop Sci., 47:285-293

Venuprasad, R., Dalid, C.O., Del Valle, M., Zhao, D., Espiritu, M., Sta Cruz M.T, Amante M, Kumar .A, Atlin G.N. (2009a). Identification and characterization of large-effect quantitative trait loci for grain yield under lowland drought stress in rice using bulk-segregant analysis. Theor. Appl. Genet., 120:177-190

Venuprasad, R., Bool, M.E., Dalid, C.O., Bernier, J., Kumar, A. and Atlin, G. N. (2009b). Genetic loci responding to two cycles of divergent selection for grain yield under drought stress in a rice breeding population. Euphytica, 167:261269

Vikram, P., Mallikarjuna Swamy, B.P, Dixit, S., Ahmed, H.U., Sta Cruz M.T., Singh, A.K and Kumar, A. (2011). qDTY1.1, a major QTL for rice grain yield under reproductive-stage drought stress with a consistent effect in multiple elite genetic backgrounds. BMC Genet., doi:10.1186/1471-2156-12-89

Vikram, P., Swamy, B.P.M., Dixit, S., Ahmed, H.. Sta Cruz, M.T., Singh, A.K., Ye, G. and Kumar, A. (2012). Bulk segregant analysis: "An effective approach for mapping consistent-effect drought grain yield QTLs in rice". Field Crops Research, 134: 185-192

Wang, S., Basten, C.J. and Zeng, Z.B. (2011). Windows QTL cartographer 2.5. department of statistics, North Carolina State University, Raleigh, NC.

Weatherley, P.E. (1950). Studies in the water relations of the cotton plant. I. The field measurements of water deficits in leaves. New Phytol, 49: 81-97 